\title{
BMJ Open Balance deficiencies in women with fibromyalgia assessed using computerised dynamic posturography: a cross-sectional study in Spain
}

\author{
Marta Pérez-de-Heredia-Torres, ${ }^{1}$ Elisabet Huertas-Hoyas, ${ }^{1}$ \\ Rosa Martínez-Piédrola, ${ }^{1}$ Domingo Palacios-Ceña, ${ }^{1}$ Jorge Alegre-Ayala, ${ }^{1}$ \\ Montserrat Santamaría-Vázquez, ${ }^{2}$ César Fernández-de-las-Peñas ${ }^{1}$
}

To cite: Pérez-de-HerediaTorres M, Huertas-Hoyas E, Martínez-Piédrola $\mathrm{R}$, et al. Balance deficiencies in women with fibromyalgia assessed using computerised dynamic posturography: a cross-sectional study in Spain. BMJ Open 2017;7:e016239. doi:10.1136/ bmjopen-2017-016239

- Prepublication history for this paper is available online. To view these files please visit the journal online (http://dx.doi org/10.1136/bmjopen-2017016239)

Received 2 February 2017 Revised 26 May 2017 Accepted 15 June 2017

\section{CrossMark}

${ }^{1}$ Department of Physical Therapy, Occupational Therapy, Physical Medicine and Rehabilitation, Universidad Rey Juan Carlos, Alcorcón, Spain

${ }^{2}$ Department of Health Sciences, Burgos Universit, Burgos, Spain

Correspondence to Elisabet Huertas-Hoyas; elisabet.huertas@urjc.es

\section{ABSTRACT}

Objectives Our aims were (1) to compare the sensory organisation of balance control and balance strategies between women with fibromyalgia (FM) and healthy women; (2) to investigate which sensory component, that is, vestibular, visual or somato-sensory, is the most affected in FM and (3) to determine the associations between the functional independence measure (FIM) and balance responses in FM.

Design Cross-sectional observational study.

Setting Urban regional hospital and university

(Universidad Rey Juan Carlos, Madrid, Spain).

Participants Twenty women with FM and 20 matched healthy women.

Primary/secondary outcome measures The sensory organisation test (SOT) was used to determine postural sway and balance during six different conditions with subjects in a standing position. The FIM was used to determine the level of functional independence in daily life activities (ADL). Between-group differences were analysed with analysis of covariance, and the Spearman's test was used for correlations.

Results Significant differences between-groups and between-conditions were found for all SOT conditions (all, $p<0.001$ ): women with FM showed lower scores being the vestibular score the most affected. Different correlations between SOT conditions and some specific ADL were observed in the FM group: bathing activity and balance condition $6\left(r_{s}=0.541 ; p<0.001\right)$, bed transfers activity and conditions $2\left(r_{s}=0.491 ; p<0.001\right)$ and $3\left(r_{s}=0.510\right.$; $p<0.001)$, positioning strategy six and dressing the upper $\left(r_{s}=0.530 ; p<0.001\right)$ or lower $\left(r_{s}=0.562 ; p<0.001\right)$ body, and toileting $\left(r_{s}=0.521 ; p<0.001\right)$ : the greater the loss of balance, the greater the interference on some daily life activities.

Conclusions Women with FM exhibited balance deficiencies and used different strategies for maintaining their balance in standing, which was associated with a negative impact on functional independence.

\section{INTRODUCTION}

Fibromyalgia (FM) is a chronic syndrome that has a considerable functional impact on
Strengths and limitations of this study

- This is the first study investigating the association between postural balance and functional interference with activity on daily living.

- The sample size was relatively small and from the same regional hospital.

- We only included women, but not men, diagnosed with fibromyalgia.

patients. It is estimated that between $10 \%$ and $15 \%$ of the general population is affected by this syndrome in Europe. ${ }^{1}$ The main complaint is generalised long-lasting muscle pain, which is typically described as deep and intense and worsening with intense physical exercise, cold and/or emotional mental stress. This widespread pain is accompanied by other symptoms including asthenia, fatigue and non-restorative sleep together with other poorly defined symptoms. ${ }^{2}$ Individuals with FM can also present muscle asymmetry ${ }^{3}$ and difficulty for relaxing the muscles, ${ }^{4}$ which can contribute to fatigue and pain, leading to posture and balance deficit. In fact, balance problems are among the most debilitating symptoms reported by patients with $\mathrm{FM}^{5}{ }^{5}$ In addition, postural disturbances affecting the vertebral column have been also found ${ }^{7}$ as well as lower spatio-temporal parameters during gait $^{8}$ and a higher risk of falls. ${ }^{9-11}$ Finally, FM can be associated with general inactivity, ${ }^{12}$ which can lead to negative effects on the functional capacity of the patient.

Postural control requires the appropriate integration of sensory, visual, vestibular and somatosensory information into the central nervous system (mainly integrated by proprioceptive and cutaneous sensitivity). Posturography is a technique that enables a quantitative assessment of postural control 
by studying the displacements of the centre of pressure in different circumstances simulating actions from normal daily life. ${ }^{13}$ This technique itself does not enable a diagnosis; however, it provides information regarding functional status and can be of value for guiding treatment.

Some of the most utilised tests in posturography include the sensory organisation test (SOT), the motor control test and the adaptation test. ${ }^{14}$ The SOT enables isolation of components from the vestibular, visual and somatosensory systems that participate in the maintenance of postural control, enabling users to determine the site of the main disorder causing the loss of balance. ${ }^{15}$ Some previous studies have reported the presence of balance and postural control deficits in women with FM using different procedures. ${ }^{16-18}$ Muto et al ${ }^{17}$ observed that patients with FM exhibited impaired postural control, for example, increased speed of oscillation of the centre of gravity and lower balance self-efficacy as assessed with the modified clinical test of sensory interaction on balance and the balance self-efficacy. In this study, impaired postural control and low balance self-efficacy were associated with pain severity and muscle strength. ${ }^{17}$ Jones $e t a l^{16}$ found that FM patients showed lower scores in almost all conditions of the SOT and an increased number of falls. In this study, postural stability was associated with related disability, cognitive impairment and body mass index, but not with medication intake, pain severity or muscle strength. ${ }^{16}$ In a pilot study using the SOT, Russek and Fulk $^{18}$ reported that $34 \%$ of FM subjects scored below the fifth percentile for population normative data in some SOT conditions. These authors also found a negative association between the somatosensory score of the SOT and FM-related disability. ${ }^{18}$ Although these studies support the occurrence of balance problems in patients with FM using the SOT, they did not investigate the association of balance disturbances with functional independence in activities of daily living (ADLs). The identification of an association between balance problems and ADL disturbances can help clinicians for developing specific therapeutic strategies for patients with FMs. To the best of the author's knowledge, no study has previously investigated this association in patients with FM.

Therefore, the aims of this study were (1) to compare sensory organisation of balance control and balance strategies between women with FM and healthy controls; (2) to investigate which sensory component (vestibular, visual or somatosensory) is the most affected in FM women and (3) to determine the potential association between the functional independence measure (FIM) and balance responses in women with FM.

\section{METHODS}

\section{Research design}

A cross-sectional study was performed. We conducted non-probabilistic sampling of consecutive cases, where subjects who met the established criteria were included.
The study was conducted during the second semester of 2015.

\section{Participants}

Advertisements were placed in local newspapers in order to recruit healthy women from the general population for acting as control group. Participants were considered as healthy controls if they reported: no spontaneous pain symptoms at the moment of the study, no history of chronic pain (lasting more than 3 months), no pain experienced during the previous year prior to the study, no pain-related diagnoses and participants who were not taking antidepressant or analgesic medication.

Women with diagnosis of FM were recruited from the Department of Rheumatology at the Hospital Fundación Alcorcón (Spain). An experienced rheumatologist confirmed the FM diagnosis based on a combination of both American College of Rheumatology (ACR) criteria $(1990 / \mathrm{m} 2010) .{ }^{19}{ }^{20}$ It has been suggested that a combination of 1990 and m2010 criteria is recommended since it had the best diagnostic features. ${ }^{21}{ }^{22}$ Tender points were tested by digital palpation at the 18 sites according to the ACR protocol. ${ }^{19}$ Participants were asked to indicate whether they experienced pain in response to a pressure of approximately $4 \mathrm{~kg}$ exerted by the examiner. ${ }^{19}$ Furthermore, the presence of fatigue, altered sleep patterns and other sensory symptoms self-perceived by the patient were recorded. ${ }^{20}$ Face-to-face structured medical interviews were performed to determine the time of the diagnosis, sociodemographic and clinical data, current medication intake and presence of psychiatric disorders.

Exclusion criteria for both groups included: (1) co-morbid medical diagnoses, for example, cardiopulmonary disorders, inflammatory disease, obesity and other diagnoses; (2) malignancy; (3) psychiatric illnesses diagnosis, for example, schizophrenia or substance abuse; (4) depression (Beck Depression Inventory-II $>8$ points); (5) previous history of surgery; (6) history of whiplash; (7) uncontrolled endocrine disorders (ie, hyperthyroidism or hypothyroidism, diabetes) or (8) pregnancy.

Participants were matched based on their age and hand dominance to gain homogeneity in the sample during the performance of those ADLs involving the upper extremity. Hand dominance was determined by self-reports regarding the hand used for writing.

\section{Ethical considerations}

This study was conducted in accordance with the ethical standards of the Declaration of Helsinki and was reviewed and approved by the Ethics committee of the Hospital Fundación Alcorcón (protocol FHA-URJC 032). All subjects provided written informed consent.

\section{Study procedure}

The study protocol for the SOT was the same for all participants. In addition, women with FM also fulfilled the Spanish version of the Fibromyalgia Impact Questionnaire $(\mathrm{FIQ})^{23}$ to assess FM-related disability. ${ }^{24}$ All participants were verbally informed of the study, accepted 
the informed consent and were familiarised with the different outcomes before starting data collection.

First, the SOT protocol was performed and subsequently participants completed the remaining assessments. All assessments were performed at a similar time of the day in the Laboratory for Movement analysis, Biomechanics, Ergonomics and Motor Control, located at the Department of Physical Therapy, Occupational Therapy, Rehabilitation and Physical Medicine, Universidad Rey Juan Carlos (Spain).

The FIM assessment took place in a suitably equipped apartment, via observation of subject's functional independence demonstrated during the performance of ADL contained in the scale. An external evaluator, blinded to the participant's condition, performed the assessments.

\section{Outcome measures}

\section{Functional independence measure}

The FIM provides an assessment of the level of functional independence in daily life activities. ${ }^{25}$ It also provides information primarily on cognitive and motor performance via 18 items. Scores range from 1 to 7 , with higher scores corresponding to a higher level of functional independence. The possible score ranges from 18 to 126 points. The FIM includes observation and face-to-face interviews. This tool has demonstrated excellent psychometric properties. ${ }^{26-28}$

\section{Sensory organisation test}

The posturography device used in this study was the SOT assessment, which belongs to the Smart Balance Master, by Neurocom EQ0501, International (Oregon, USA).$^{29} 30$ The device consists of a platform connected to symmetrically placed transducers measuring the vertical and horizontal shear forces exercised through the anterior=posterior axis in the plane parallel to the floor. It is also equipped with a mobile visual surround screen. Both the visual surround and platform are computer-controlled and can move simultaneously. This device was connected to a PC Pentium I, with Smart Balance Master V.5.0 software and a Samsung monitor. The reports obtained for each participant were saved on the computer's hard drive.

In order to conduct the SOT, an individual's postural sway, and thereby balance, is measured under six different conditions during standing. During these tests, the base of support and the visual surround screen can move according to the patient's balancing responses and the strategy used for maintaining an upright position. For instance, no altered stimuli are given in condition 1 , whereas visual information is removed in condition 2 by asking the participant to close their eyes. In condition 3, the visual surround is moving with the subject's anterior-posterior body sway, whereas in condition 4, the platform rotates with the subject's anterior=posterior body sway. In condition 5, subjects close their eyes and the platform moves with the subject anterior=posterior body sway. Finally, in condition 6 , the visual screen and the platform are moved with the subject's anterior=posterior body sway. Briefly, the six conditions can be resumed as follows: (1) eyes open, fixed surround and support platform; (2) eyes closed, fixed surround and support platform; (3) eyes open, moving surround (moving proportional to the angle of anterior=posterior body sway) and fixed support platform; (4) eyes open, fixed surround and moving support platform (moving proportional to the angle of anterior-posterior body sway); (5) eyes closed, fixed surround and moving support platform and (6) eyes open, moving surround and support platform. Tests were always performed following these steps in order. Each condition was performed three consecutive times and the mean was considered in the analysis. In total, the duration of the tests lasted approximately $12 \mathrm{~min}$ for each patient; therefore, it can be considered a non-fatiguing assessment. This procedure has shown good test_retest reliability in healthy people. ${ }^{31}$

Participants were encouraged to maintain their stability and centre of gravity, despite the movement of the visual surround or the base of support. The participant's centre of gravity was displayed on the upper half of the screen. The feet were correctly positioned facing the visual surround during the entire test. If the participant fell, took a step or touched the visual surround, the test was interrupted and the fall was registered. Data assessments were performed automatically and compared with theoretical normative electronic data. The score of each condition consist of a percentage that compares the subject anterior-posterior centre of pressure sway with the theoretical limits of stability. The score is registered on a bar chart ranging from $0 \%$ to $100 \%$ where $0 \%$ represents the least stable (fall) and $100 \%$ indicates perfect stability. ${ }^{29}$

In addition, combination of the results obtained in the different conditions provides a ratio score of each sensory system (somatosensory, vestibular or visual). The somatosensory ratio (condition 2/condition 1 ) determines how successfully a person uses input from the somatosensory system for balance; the visual ratio (condition 4/condition 1) determines how successfully a person uses visual system for balance and the vestibular ratio (condition 5/ condition 1) determines how successfully a person uses input from the vestibular system for balance.

Finally, a strategy score for each SOT condition is also calculated with scores near 100 indicating the use of an ankle strategy and scores near 0 indicating a hip strategy.

\section{Sample size calculation}

The sample size was calculated using the Ene V.3.0 software (Autonomic University of Barcelona, Spain). The sample calculation was based on detecting significant moderate correlations $(\mathrm{r}=0.60)$ between the SOT conditions and FIM variables with an alpha level $(\alpha)$ of 0.05 , and a desired power $(\beta)$ of $80 \%$. This generated a sample size of at least 19 subjects.

\section{Statistical analysis}

The SPSS statistical package was used for data analysis (V.19.0, SPSS, Chicago, Illinois, USA). The Kolmogorov-Smirnov test was used to analyse the normal distribution of the variables $(p>0.05)$. Quantitative data without a 
normal distribution (clinical data and FIM scores) were analysed with non-parametric tests and those data with a normal distribution (SOT conditions) were analysed with parametric tests. A $2 \times 6$ analysis of covariance (ANCOVA) with group (FM or controls) as a between-subjects factor and with condition of the SOT (from 1 to 6 ) as a within-subjects factor and body mass index as covariate were used to analyse differences in the assessments of balance responses and strategies used for maintaining the upright position in the SOT. The main hypothesis of interest was the group $\times$ condition interaction. Furthermore, unpaired Student t-tests were also conducted to determine between-groups difference for the ratio score of each sensory system (somatosensory, vestibular or visual). Finally, the Spearman's rho $\left(r_{s}\right)$ test was used to analyse potential associations between the clinical variables related to symptoms, disability, FIM and SOT conditions in the FM group. The statistical analysis was generally conducted at a $95 \%$ significance level; but, we corrected for multiple comparisons using the Holm-Bonferroni adjustment, ${ }^{32}$ assuming a significant alpha level of 0.008 (six independent-samples t-tests by SOT condition).

\section{RESULTS}

\section{Demographic and clinical data}

Twenty-nine $(n=29)$ women with FM were screened for eligibility criteria between January and November 2015. Nine women were excluded as follows: previous surgery $(n=3)$, whiplash syndrome $(n=2)$, pregnancy $(n=2)$, diabetes $(\mathrm{n}=1)$ and litigation $(\mathrm{n}=1)$. The final sample consisted of 20 women with FM, aged 35-55 years (mean: $48 \pm 6$ years), who satisfied all the eligibility criteria and agreed to participate. In addition, 20 matched healthy women, aged $35-56$ years (mean: $47 \pm 6$ years), were also included. There were no significant differences in age $(\mathrm{p}=0.909)$ or body mass index (control: 23.8 \pm 1.3 ; FM: $24.2 \pm 1.5, \mathrm{p}=0.508$ ) between both the groups. All participants were right-handed. Seventeen $(85 \%)$ women with
FM $(85 \%)$ were regularly taking non-steroidal anti-inflammatory medications. The FIQ revealed a moderate disability with a mean score of 57.9 (95\% CI 53.1 to 62.6). All participants completed all assessments and there were no missing data.

\section{Fibromyalgia and SOT}

The ANCOVA revealed significant differences between groups $(F=21.634 ; \mathrm{p}<0.001)$ and conditions $(F=45.164$; $\mathrm{p}<0.001)$ for the balance responses on the SOT: women with FM displayed significantly $(\mathrm{p}=0.005)$ lower values in all SOT conditions than healthy women and scores of conditions 4-6 were significantly lower (all, $\mathrm{p}<0.01$ ) than those for conditions 1-3 (table 1). A significant group $\times$ condition interaction was also found $(F=3.404$; $\mathrm{p}=0.006)$ : differences between conditions 4-6 scores and conditions 1-3 were significantly more pronounced within the FM group. No effect of the body mass index was observed.

We found significant $(\mathrm{t}=2.901 ; \mathrm{p}=0.006)$ lower vestibular ratio score in women with FM (mean: $0.55 \pm 0.2$ ) when compared with healthy women (mean: $0.72 \pm 0.15$ ). No significant differences in somatosensory $(t=0.011$; $\mathrm{p}=0.989)$ and visual $(\mathrm{t}=1.900 ; \mathrm{p}=0.065)$ ratios between women with FM (somatosensory: $0.95 \pm 0.03$; visual: $0.82 \pm 0.15$ ) and healthy women (somatosensory: $0.96 \pm 0.03$; visual: $0.90 \pm 0.1$ ) were observed.

The ANCOVA also revealed significant betweengroups $(F=10.456 ; \quad \mathrm{p}<0.001)$ and between-conditions $(F=35.301 ; \mathrm{p}<0.001)$ differences for the balance strategies used during the SOT conditions: FM women displayed significantly lower values $(p<0.001)$ in all conditions than healthy women (table 1), suggesting a greater use of the hip instead of the ankle. Again, scores on conditions 4-6 were lower than those values for conditions 1-3 $(\mathrm{p}<0.001)$. No significant groupxcondition interaction $(F=1.170 ; \mathrm{p}=0.325)$ or effect of the body mass index was observed.

Table 1 Differences in the values of the SOT between women with FM and healthy women

\begin{tabular}{|c|c|c|c|c|c|c|}
\hline & Condition $1^{*}$ & Condition $2^{*}$ & Condition $3^{*}$ & Condition $4^{\star} \dagger$ & Condition $5^{\star} \dagger$ & Condition $6^{*} \dagger$ \\
\hline \multicolumn{7}{|l|}{ Balance } \\
\hline Women with FM & $93.1 \pm 5.4$ & $89.7 \pm 4.9$ & $86.3 \pm 7.0$ & $76.5 \pm 13.4$ & $52.1 \pm 18.0$ & $54.7 \pm 19.4$ \\
\hline \multicolumn{7}{|l|}{ Strategy } \\
\hline Women with FM & $98.5 \pm 1.6$ & $97.3 \pm 4.4$ & $96.7 \pm 3.4$ & $85.7 \pm 8.1$ & $76.1 \pm 12.0$ & $78.1 \pm 7.6$ \\
\hline
\end{tabular}

Data are expressed as means \pm SD. Condition 1: eyes open, fixed visual surround and fixed support platform; condition 2: eyes closed, fixed support platform; condition 3: eyes open, mobile visual surround (moving proportional to the angle of anterior-posterior body sway) and the fixed support platform; condition 4: eyes open, fixed visual surround and mobile support platform (moving proportional to the angle of anterior-posterior body sway); condition 5: eyes closed, mobile support platform and condition 6: eyes open, mobile visual surround and mobile support platform.

*Statistically significant differences between patients and controls $(p<0.001$; ANCOVA test). †Statistically significant differences between conditions 1-3 ( $p<0.01$; ANCOVA test). ANCOVA, analysis of covariance; FM, fibromyalgia; SOT, sensory organisation test. 
Table 2 Correlations between the clinical pain variables and the SOT balance values in women with FM

\begin{tabular}{lllll} 
& Duration of symptoms & Current pain & Best pain & Worse pain \\
\hline Condition 1 & $r_{s}=0.124 ; p=0.602$ & $r_{s}=0.276 ; p=0.239$ & $r_{s}=0.242 ; p=0.304$ & $r_{s}=0.169 ; p=0.476$ \\
Condition 2 & $r_{s}=0.291 ; p=0.213$ & $r_{s}=0.051 ; p=0.830$ & $r_{s}=0.334 ; p=0.149$ & $r_{s}=0.179 ; p=0.450$ \\
Condition 3 & $r_{s}=0.310 ; p=0.183$ & $r_{s}=0.152 ; p=0.552$ & $r_{s}=0.131 ; p=0.581$ & $r_{s}=0.127 ; p=0.593$ \\
Condition 4 & $r_{s}=0.308 ; p=0.186$ & $r_{s}=0.084 ; p=0.736$ & $r_{s}=0.076 ; p=0.749$ & $r_{s}=0.07 ; p=0.769$ \\
Condition 5 & $r_{s}=0.135 ; p=0.571$ & $r_{s}=0.111 ; p=0.642$ & $r_{s}=0.151 ; p=0.526$ & $r_{s}=0.219 ; p=0.354$ \\
Condition 6 & $r_{s}=0.123 ; p=0.606$ & $r_{s}=0.050 ; p=0.835$ & $r_{s}=0.156 ; p=0.511$ & $r_{s}=0.156 ; p=0.512$ \\
\hline
\end{tabular}

FM, fibromyalgia; $r_{s}$, Spearman's correlation test (Spearman's rho); SOT, sensory organisation test.

\section{Correlations between clinical variables and SOT conditions in FM}

Within the group of women with FM, no significant correlation was found between the duration (years) of neither pain nor the intensity of the symptoms with any of the SOT conditions. Table 2 displays correlation coefficients and the statistical significance for all conditions in the balance section, whereas table 3 displays the same data for each condition within the strategy section.

\section{Correlations between functionality and SOT conditions}

Positive correlations between different SOT conditions and different ADLs variables were found in the group of women with FM (table 4). The balance condition 6 (eyes open, mobile visual surround-mobile platform) was moderately associated with bathing activity $\left(r_{s}=0.541\right.$; $\mathrm{p}<0.001$ ), whereas conditions 2 and 3 were positively and moderately associated with bed transfers activity $\left(r_{\mathrm{s}}=0.491\right.$; $\mathrm{p}<0.001$ and $\mathrm{r}_{\mathrm{s}}=0.510 ; \mathrm{p}<0.001$, respectively): the lower the score balance in these conditions, the poorer the function in the respective ADLs.

Similarly, significant positive correlations were found between positioning strategy number 6 and the following ADLs: dressing upper body $\left(\mathrm{r}_{\mathrm{s}}=0.530 ; \mathrm{p}<0.001\right)$, dressing the lower body $\left(r_{s}=0.562 ; \mathrm{p}<0.001\right)$ and toileting $\left(r_{s}=0.521\right.$; $\mathrm{p}<0.001)$ : the worse the balance strategy, the greater interference with functional independence in these ADL.

\section{DISCUSSION}

This study found that women with FM exhibit worse balance scores compared with healthy women as assessed with the SOT, which are in agreement with previous studies. ${ }^{1618}$ In fact, differences were higher with the eyes closed and moving surrounding surfaces. Furthermore, the strategy used for stabilising the ankle joint was poor in women with FM. Nevertheless, the most significant contribution of this study was the association of balance scores with functional independence during ADL.

Women with FM exhibited lower scores in all SOT conditions compared with healthy women suggesting poor balance. Our results agree with those previously observed by Jones $e t a l^{16}$ and Russek and Fulk ${ }^{18}$ who also reported significantly lower scores in all SOT conditions in individuals with FM. It is interesting to note that the scores observed in our study were similar to those reported in these previous studies. ${ }^{1618}$ Current and previous evidence would suggest that subjects with FM exhibit poor general balance when compared with healthy women. Nevertheless, although all SOT conditions showed lower scores in FM, the vestibular ratio was the most significantly impaired in our sample of women with FM. This may be related to the fact that scores in the last SOT conditions (4-6) were significantly much lower in the FM group than in the healthy group. As previously suggested, lower scores in conditions 4-6 compared with conditions $1=3$ suggest a degree of somatosensory dependence. ${ }^{15}$ This hypothesis is in line with the study by De Brujin et $a l^{33}$ who found that balance in patients with FM was more optimal on firm and regular surfaces. In fact, Russek and Fulk ${ }^{18}$ and this study did not find significant differences within the somatosensory system ratio between individuals with FM and healthy people, suggesting that it is the vestibular, and probably the visual, system ${ }^{16}$ the most affected in this population.

To determine the mechanisms related to poor balance in patients with FM is beyond the scope of this study, but

Table 3 Correlations between the clinical pain variables and the SOT strategy values in women with FM

\begin{tabular}{lllll}
\hline & Duration of symptoms & Current pain & Best pain & Worse pain \\
\hline Condition 1 & $r_{s}=-0.088 ; p=0.713$ & $r_{s}=0.076 ; p=0.749$ & $r_{s}=0.204 ; p=0.388$ & $r_{s}=0.047 ; p=0.846$ \\
Condition 2 & $r_{s}=0.124 ; p=0.602$ & $r_{s}=-0.032 ; p=0.894$ & $r_{s}=-0.399 ; p=0.082$ & $r_{s}=-0.210 ; p=0.613$ \\
Condition 3 & $r_{s}=0.123 ; p=0.604$ & $r_{s}=-0.073 ; p=0.759$ & $r_{s}=-0.040 ; p=0.867$ & $r_{s}=-0.022 ; p=0.926$ \\
Condition 4 & $r_{s}=-0.069 ; p=0.772$ & $r_{s}=-0.161 ; p=0.498$ & $r_{s}=-0.118 ; p=0.621$ & $r_{s}=-0.130 ; p=0.585$ \\
Condition 5 & $r_{s}=0.036 ; p=0.879$ & $r_{s}=0.046 ; p=0.848$ & $r_{s}=-0.140 ; p=0.555$ & $r_{s}=0.380 ; p=0.098$ \\
Condition 6 & $r_{s}=-0.097 ; p=0.685$ & $r_{s}=-0.086 ; p=0.718$ & $r_{s}=-0.010 ; p=0.966$ & $r_{s}=-0.151 ; p=0.525$ \\
\hline
\end{tabular}

FM, fibromyalgia; $r_{s}$, Spearman's correlation test (Spearman's rho); SOT, sensory organisation test. 


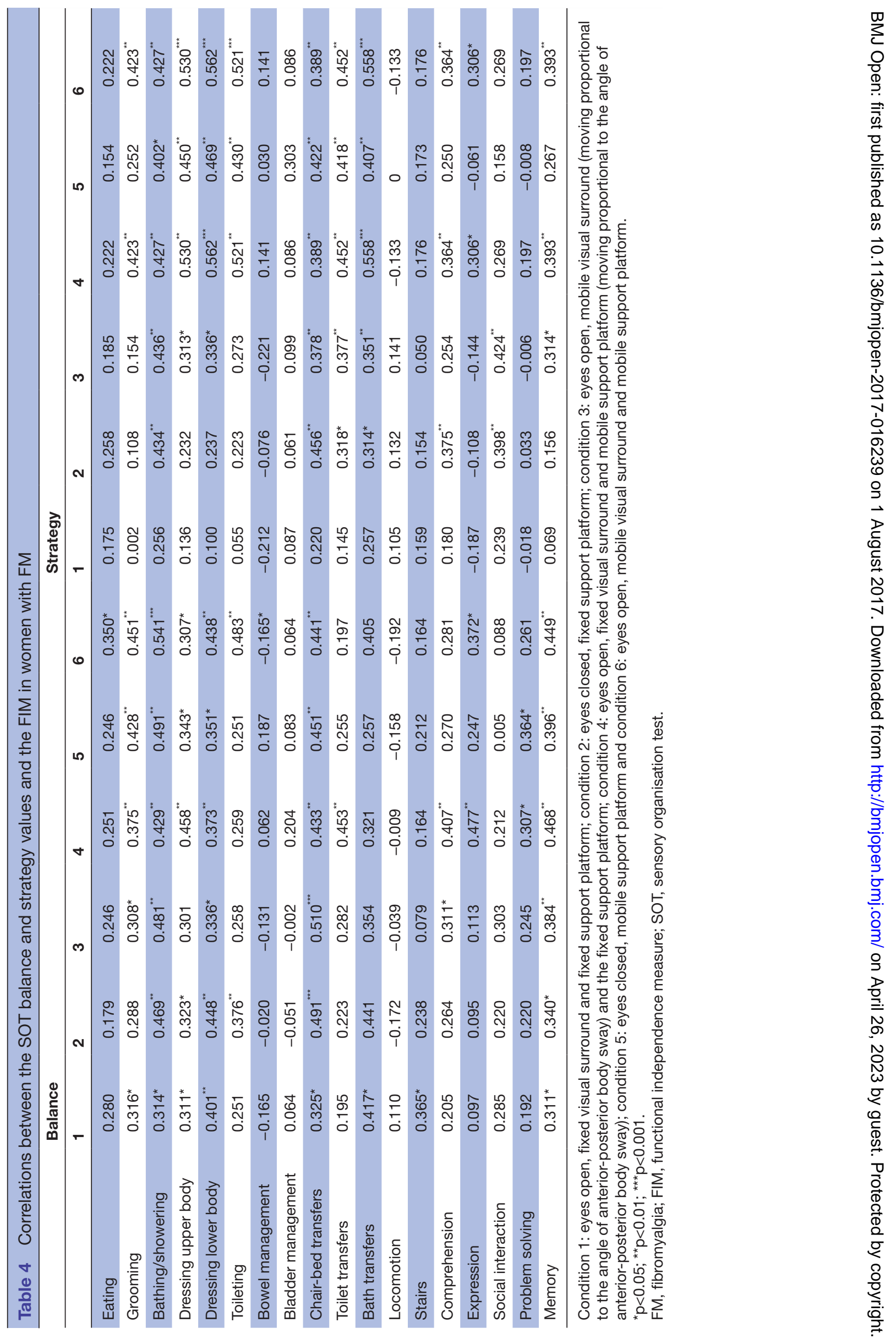


some hypotheses have been proposed. As FM is characterised by abnormal nociceptive processing, it is possible that multiple processing disturbances may lead to poor balance. In addition, other processing abnormalities of the central nervous system, for example, cognitive dysfunction, could also contribute to postural instability. In fact, Bayazit et $a l^{34}$ suggested that women with FM have neural brainstem disintegration, which could lead to abnormal perception of audiovestibular inputs and to abnormal auditory brainstem response. Current and previous finding demonstrating that the vestibular system was the most affected in individuals with FM would support this hypothesis. Nevertheless, as we did not specifically evaluate the function of the vestibular system in our sample of women with FM, the current results do not permit to determine whether the low scores on the vestibular component of the SOT were due to peripheral or central deficits.

In addition to lower balance scores, we also observed that our sample of women with FM also used different strategy than healthy women for maintaining their balance. The SOT strategy scores indicate that woman with FM use a hip strategy to maintain their balance, whereas healthy women use a more ankle strategy. Some possible reasons for these changes in balance strategy can be the presence of muscle trigger points in the gastrocnemius and tibialis anterior muscles ${ }^{16}$ or the greater muscle fatigue in the tibialis anterior muscle ${ }^{35}$ observed in FM. Future studies should investigate neurophysiological mechanisms related to changes in balance strategy in subjects with FM.

The most relevant result of our study was the positive association between balance scores and functional independence during ADL because the greater the loss of postural balance, the greater the interference with those ADL activities requiring proper postural control and balance, for example, bathing and dressing. These findings are valuable for planning proper treatment interventions because deficits or loss of independence in these ADLs has a negative impact on the quality of life of the patients. Our results agree with the study by Amris et $a \vec{l}^{6}$ who investigated women with widespread chronic pain symptoms and observed that patients with FM have substantial problems affecting their daily life and are liable to need community support. Therefore, current findings can help for planning multidisciplinary interventions for individuals with FM. For instance, balance strategies and postural control can be treated with physical therapy, whereas therapeutic strategies for improvement of ADL efficacy should be applied by occupational therapists. Furthermore, cognitive behaviours or fear to movement can be benefited from psychological treatment.

Finally, this study presents several limitations. First, although significant differences were found between groups, these were based on a small sample size. Nevertheless, we believe that a large sample size would not alter the direction of our findings. Furthermore, the population included was recruited from a regional hospital, which makes generalisation of the results to the general population difficult. Consequently, further epidemiological studies with larger sample sizes are needed to enable a more generalised interpretation of the results. Second, we analysed around 264 correlations in our study. It is possible that a type I error would be present. A greater sample size would help to elucidate if the significant association observed in this study are further significant or not. Third, we only included women diagnosed with FM. It is unknown whether men with FM would also exhibit similar results. Fourth, we excluded women with FM and co-morbid depressive symptoms, hence extrapolation of our results to this subgroup of patients with FM should be considered with caution. Although it seems that depression or anxiety may affect balance, ${ }^{37}$ we do not know the effect of depression in the outcomes included in our study, particularly those related to the FIM. Fifth, as fatigue is a common denominator in individuals with FM, it was unknown whether the inclusion of several functional outcomes could be affected by rest-periods.

\section{CONCLUSIONS}

Women with FM exhibit poor balance and use different strategies for maintaining upright posture when compared with healthy women, which may be associated with disturbances of the vestibular system. In addition, balance deficits are associated with a negative impact on functional independence in ADL. Multidisciplinary treatments directed at improving the problems faced during ADLs may help improve the autonomy of women with FM.

Contributors Concept development (provided idea for the research): MP. Design (plane the methods to generate the results): MP, EH, RM, DP, JA, MS, CF. Supervision (provided oversight, responsible for organization and implementation, writing of the manuscript): MP, EH and CF. Data collection/processing (responsible for experiments, patient management, organization, or reporting data): RM, DP, and MS. Analysis/interpretation (responsible for statistical analysis, evaluation, and presentation of the results): MP, EH, DP, JA, CF. Literature search (performed the literature search): MP, EH, RM, DP, and MS. Writing (responsible for writing a substantive part of the manuscript): MP, EH, RM, DP, JA, MS, CF. Critical review (revised manuscript for intellectual content, this does not relate to spelling and grammar checking): MP, EH and CF.

Funding This research received no specific grant from any funding agency in the public, commercial, or not for profit sectors.

Competing interests None declared.

Patient consent Obtained

Ethics approval Human Subjects Committee Approval Letter HUFA-URJC 032.

Provenance and peer review Not commissioned; externally peer reviewed.

Data sharing statement Extra data can be accessed via the Dryad data repository at http://datadryad.org/ with the doi:10.5061/dryad.3j6rr.

Open Access This is an Open Access article distributed in accordance with the Creative Commons Attribution Non Commercial (CC BY-NC 4.0) license, which permits others to distribute, remix, adapt, build upon this work non-commercially, and license their derivative works on different terms, provided the original work is properly cited and the use is non-commercial. See: http://creativecommons.org/ licenses/by-nc/4.0/

(C) Article author(s) (or their employer(s) unless otherwise stated in the text of the article) 2017. All rights reserved. No commercial use is permitted unless otherwise expressly granted. 


\section{REFERENCES}

1. Branco JC, Bannwarth B, Failde I, et al. Prevalence of Fibromyalgia: a survey in five European countries. Semin Arthritis Rheum 2010;39:448-53.

2. Villanueva VL, Valía JC, Cerdá G, et al. Fibromyalgia: diagnosis and treatment. current knowledge. Rev Soc Española Dolor 2004;11:430-43.

3. Mitani Y, Fukunaga M, Kanbara K, et al. Evaluation of psychophysiological asymmetry in patients with Fibromyalgia syndrome. Appl Psychophysiol Biofeedback 2006;31:217-25.

4. Westgaard $\mathrm{RH}$. Muscle activity as a releasing factor for pain in the shoulder and neck. Cephalalgia 1999;19 Suppl 25:1-8.

5. Bennett RM, Jones J, Turk DC, et al. An internet survey of 2,596 people with Fibromyalgia. BMC Musculoskelet Disord 2007;8:27.

6. Katz R, Ferbert S, Leavitt F, et al. Fibromyalgia patients report many symptoms other than pain and fatigue. Arthritis Rheum 2007:56:1532.

7. Müller W, Kelemen J, Stratz T, et al. Spinal factors in the generation of Fibromyalgia syndrome. Z Rheumatol 1998;57 Suppl 2:S36-S42.

8. Latorre-Román P, Santos-Campos M, Heredia-Jimenez J, et al. Analysis of the performance of women with Fibromyalgia in the six-minute walk test and its relation with health and quality of life. $J$ Sports Med Phys Fitness 2014;54:511-7.

9. Meireles SA, Antero DC, Kulczycki MM, et al. Prevalence of falls in Fibromyalgia patients. Acta Ortop Bras 2014;22:163-6.

10. Jones KD, Horak FB, Winters-Stone $\mathrm{K}$, et al. Fibromyalgia is associated with impaired balance and falls. J Clin Rheumatol 2009;15:16-21.

11. Akkaya N, Akkaya S, Atalay NS, et al. Assessment of the relationship between postural stability and sleep quality in patients with Fibromyalgia. Clin Rheumatol 2013;32:325-31.

12. Simms RW, Roy SH, Hrovat M, et al. Lack of association between Fibromyalgia syndrome and abnormalities in muscle energy metabolism. Arthritis Rheum 1994;37:794-800.

13. Galeano D, Brunetti F, Torricelli D, et al. A tool for balance control training using muscle synergies and multimodal interfaces. Biomed Res Int 2014;2014:1-13.

14. Leitner C, Mair P, Paul B, et al. Reliability of posturographic measurements in the assessment of impaired sensorimotor function in chronic low back pain. J Electromyogr Kinesiol 2009;19:380-90.

15. Posturografía BR. En: bartual J, Pérez N (eds). El Sistema vestibular y Sus alteraciones. Barcelona, Masson 1988:150-5.

16. Jones KD, King LA, Mist SD, et al. Postural control deficits in people with Fibromyalgia: a pilot study. Arthritis Res Ther 2011;13:R127.

17. Muto L, Mango P, Sauer J, et al. Postural control and balance self efficacy in women with Fibromyalgia. Are there differences? Eur J Physl Rehabil Med 2015;51:149-54.

18. Russek LN, Fulk GD. Pilot study assessing balance in women with Fibromyalgia syndrome. Physiother Theory Pract 2009;25:555-65.

19. Wolfe F, Smythe HA, Yunus MB, et al. The American College of Rheumatology 1990 Criteria for the classification of Fibromyalgia. Report of the Multicenter Criteria Committee. Arthritis Rheum 1990;33:160-72

20. Wolfe F, Clauw DJ, Fitzcharles M-A, et al. The American College of Rheumatology Preliminary Diagnostic Criteria for Fibromyalgia and Measurement of Symptom Severity. Arthritis Care Res 2010;62:600-10.

21. Segura-Jiménez V, Aparicio VA, Álvarez-Gallardo IC, et al. Validation of the modified 2010 American College of Rheumatology diagnostic criteria for Fibromyalgia in a Spanish population. Rheumatology 2014;53:1803-11.

22. Segura-Jiménez V, Soriano-Maldonado A, Álvarez-Gallardo IC, et al. Subgroups of Fibromyalgia patients using the 1990 American College of Rheumatology criteria and the modified 2010 preliminary diagnostic criteria: the al-Ándalus project. Clin Exp Rheumatol 2016;34:S26-33.

23. Esteve-Vives J, Rivera Redondo J, Isabel Salvat Salvat M, et al. [Proposal for a consensus version of the Fibromyalgia Impact Questionnaire (FIQ) for the Spanish population]. Reumatol Clin 2007;3:21-4.

24. Burckhardt CS, Clark SR, Bennett RM, et al. The Fibromyalgia impact questionnaire: development and validation. J Rheumatol 1991;18:728-33.

25. Guide for the Uniform Data set for Medical Rehabilitation (Adult FIM). Version 4.0. Buffalo (Nueva York): State University of New York at Buffalo, 1993.

26. Dodds TA, Martin DP, Stolov WC, et al. A validation of the functional independence measurement and its performance among rehabilitation inpatients. Arch Phys Med Rehabil 1993;74:531-6.

27. Heinemann AW, Linacre JM, Wright BD, et al. Prediction of rehabilitation outcomes with disability measures. Arch Phys Med Rehabil 1994;75:133-43.

28. Hobart JC, Lamping DL, Freeman JA, et al. Evidence-based measurement: which disability scale for neurologic rehabilitation? Neurology 2001;57:639-44.

29. Nashner LM. Computerized dynamic posturography. In: Jacobson GP, Newman CW, Kartush JM, eds. Handbook of balance function and testing. St. Louis: Mosby Year Book, 1993:280-307.

30. International N. Neurocom system operator's manual. Clackamas, OR: NeuroCom International, 2000.

31. Wrisley DM, Stephens MJ, Mosley S, et al. Learning effects of repetitive administrations of the sensory organization test in healthy young adults. Arch Phys Med Rehabil 2007;88:1049-54.

32. Holm S. A simple sequentially rejective multiple test procedure. Scand J Stat 1979;6:65-70.

33. de Bruijn ST, van Wijck AJ, Geenen R, et al. Relevance of physical fitness levels and exercise-related beliefs for self-reported and experimental pain in Fibromyalgia: an explorative study. $J$ Clin Rheumatol 2011;17:295-301.

34. Bayazit YA, Gürsoy S, Ozer E, et al. Neurotologic manifestations of the Fibromyalgia syndrome. J Neurol Sci 2002;196:77-80.

35. Bazzichi L, Dini M, Rossi A, et al. Muscle modifications in fibromyalgic patients revealed by surface electromyography (SEMG) analysis. BMC Musculoskelet Disord 2009;10:36.

36. Amris K, Wæhrens EE, Jespersen A, et al. Observation-based assessment of functional ability in patients with chronic widespread pain: a cross-sectional study. Pain 2011;152:2470-6.

37. Hassett AL, Simonelli LE, Radvanski DC, et al. The relationship between affect balance style and clinical outcomes in Fibromyalgia. Arthritis Rheum 2008;59:833-40. 